\title{
Mimic of the Cellular Antioxidant Defense System for a Sustainable Regeneration of Nicotinamide Adenine Dinucleotide (NAD)
}

\author{
Seong-Min Jo, Kai A. I. Zhang, Frederik R. Wurm,* and Katharina Landfester*
}

Cite This: ACS Appl. Mater. Interfaces 2020, 12, 25625-25632

Read Online

ABSTRACT: The prolonged use of enzymes under oxidative stress is a major challenge in enabling effective enzymatic reaction pathways. Herein, we report a biomimetic antioxidant defensive strategy capable of providing adequate protection of enzymes against superoxidemediated oxidation. Superoxide dismutase (SOD) and catalase (CAT) were chosen as scavengers and covalently encapsulated into silica nanoreactors, together with glucose dehydrogenase $(\mathrm{GDH})$, which simultaneously should produce the coenzyme nicotinamide adenine dinucleotide ( $\mathrm{NADH}$, reduced form). By the enzymatic reactions of SOD and CAT, the interior of silica nanoreactors becomes a "ROS safe zone" to protect the glucosedependent $\mathrm{NADH}$ production of coencapsulated $\mathrm{GDH}$. We further combined this protected $\mathrm{NADH}$-producing module with photocatalytic nanoparticles that enable the light-triggered oxidation of $\mathrm{NADH}$ back to $\mathrm{NAD}^{+}$(oxidized form). In combination, these two modules allow interconversion between $\mathrm{NAD}^{+}$and $\mathrm{NADH}$ by the addition of glucose or by light irradiation (LED lamp or sunlight). This protection and regeneration strategy is a versatile tool for enzyme applications for biological reactors, catalysis, or prototypes of artificial organelles or building blocks that contains fragile biomolecules and rely on the coenzyme $\mathrm{NAD}^{+} / \mathrm{NADH}$.

KEYWORDS: silica, NAD regeneration, biomimetic nanoreactors, cell mimics, antioxidant nanoreactors

\section{INTRODUCTION}

A high level of reactive oxygen species (ROS) causes severe damages to live cells due to their high reactivity. ${ }^{1}$ ROS can lead to fragmentation of polypeptide chains and alternation of electrical charge followed by conformational changes and intraor intermolecular cross-linking of proteins. ${ }^{1}$ Other cellular components (e.g., lipids and nucleotides) are also oxidized by ROS, leading to mutations or cell death. ${ }^{2}$ Against such oxidative stress, the cell has developed an enzymatic antioxidant defense strategy. Superoxide dismutase (SOD), catalase (CAT), and glutathione peroxidase are major components of the intracellular antioxidant defense system. ${ }^{3}$ These scavengers inside the cells react with both endogenous and exogenous ROS to prevent oxidative damages. It is, therefore, one of the key features of living cells. ${ }^{3}$ Inspired by the features, we developed biomimetic nanoreactors mimicking the antioxidant defense. Similar to the natural antioxidant defense system, we utilized SOD and CAT and loaded those into synthetic nanoreactors, which are semipermeable for the substrates of the enzymes but effectively retain and protect the proteins in their interior. Such artificial antioxidant defense strategies were able to generate a "ROS safe-zone" that was able to protect other enzymes from ROS-mediated damage.

In previous studies, metal catalysts (e.g., $\mathrm{MnO}_{2}, \mathrm{CeO}_{2}, \mathrm{~V}_{2} \mathrm{O}_{5}$, and $\mathrm{Pt}$ ) and ROS-responsive polymeric materials had been proposed as artificial antioxidant defense systems. ${ }^{4-9}$ However, low total turnover numbers (TTNs), toxicity, or low selectivity are major hurdles. In contrast, installing selevtive enzymes could lead to high total turnover numbers. SOD and CAT are capable of scavenging superoxide radicals and although encapsulated SOD or CAT had been reported previously, ${ }^{10-15}$ a coencapsulation with additional metabolic enzymes and protected them through antioxidant defensive functions have not been explored to date.

Regeneration of nicotinamide adenine dinucleotide (NAD) is a crucial task in synthetic biology and the development of artificial photosynthesis. ${ }^{16-20}$ The $\mathrm{NAD}$ redox pair $\left(\mathrm{NAD}^{+} /\right.$ $\mathrm{NADH}$ ) is a coenzyme that plays a crucial role in the oxidoreductase metabolism. ${ }^{18,19,21}$ Photocatalysis and enzyme reactions are emerging technologies for numerous redox reactions, including $\mathrm{NAD}$ regeneration in modern systems chemistry. ${ }^{16,20,22-28}$ During photocatalysis, ROS (e.g., singlet oxygen $\left({ }^{1} \mathrm{O}_{2}\right)$ or superoxide $\left.\left(\mathrm{O}_{2}{ }^{-}\right)\right)$are generated. Thus, a combination of photocatalysis and enzymatic metabolisms is challenging because of the denaturation of the enzymes by the produced ROS. Without additional antioxidant defense strategies, i.e., by enzymes such as SOD and CAT,

Received: March 25, 2020

Accepted: May 7, 2020

Published: May 8, 2020 


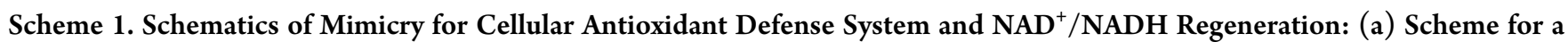
Natural Antioxidant Defense System; (b) Mimicry of the Defense Systems with NADH Regeneration ${ }^{a}$; (c) Interconversion of $\mathrm{NAD}^{+}$and $\mathrm{NADH}$ Triggered by Different Alternating Signals, Glucose, and Visible Light

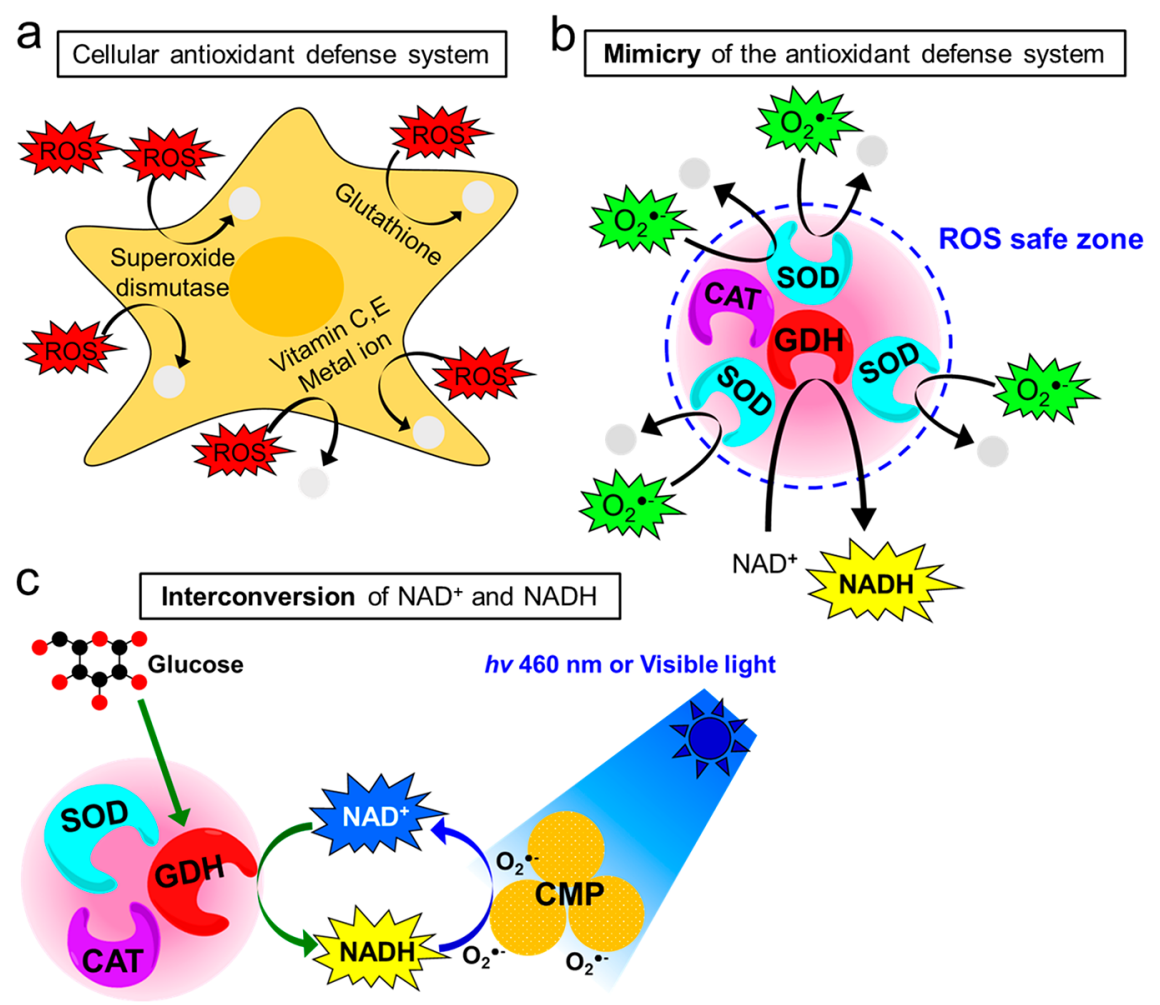

${ }^{a} \mathrm{GDH}, \mathrm{SOD}$, and CAT co-loaded into nanoreactors for the protection of enzymes (GDH) against superoxide stress and NADH regeneration. .

enzymatic/photocatalytic hybrid systems did not show the efficient and sustainable regeneration of $\mathrm{NAD}^{+}$and $\mathrm{NADH}$. We recently developed photocatalytic nanoparticles based on conjugated microporous polymers (CMPs) that catalyzed the oxidation of $\mathrm{NADH}$ to $\mathrm{NAD}^{+}$through a visible-light-triggered production of superoxide. ${ }^{29}$ By combining glycerol dehydrogenase with CMPs, oxidation of $\mathrm{NADH}$ to $\mathrm{NAD}^{+}$was successfully realized, but the enzyme could be damaged by the generated superoxide and singlet oxygen. Another hybrid system for photo-oxidation and enzymatic regeneration based on enzyme $/ \mathrm{TiO}_{2}$ was reported recently, but again, no protection of the enzymes was reported, and therefore only low amounts of $\mathrm{NAD}^{+}$regeneration were obtained. ${ }^{30}$ Thus, it is highly desired to develop an antioxidant defensive strategy to prevent oxidative damage to increase the lifetime of enzymes in such systems.

Here, we present nanoreactors loaded with SOD and CAT as a cell-mimicking antioxidant defense system together with glucose dehydrogenase (GDH) acting as additional $\mathrm{NADH}$ producing enzyme (Scheme 1). As SOD eliminates superoxide (eq 1), it can protect the NADH-producing enzymes (GDH). CAT degrades hydrogen peroxide as endogenous oxidative stress, which is a byproduct of SOD reaction (eq 2). The combination of these two enzymes guarantees the production of NADH by consuming $\mathrm{NAD}^{+}$and glucose by GDH (eq 3 ). The three enzymes were coencapsulated into a silica nanoreactor. By a sol-gel process in microemulsion, we could prepare silica nanoreactors that retained the enzymes in their interior, but possessed a semipermeable matrix for the enzyme substrates and products. To generate a $\mathrm{NAD}^{+} / \mathrm{NADH}$ regeneration system, which could produce either $\mathrm{NAD}^{+}$or
$\mathrm{NADH}$, we further combined the (GDH/SOD/CAT)-loaded nanoreactors with photocatalytic nanoparticles (CMP-NPs) (Scheme 1c).

Although $\mathrm{GDH}$ reduces $\mathrm{NAD}^{+}$to $\mathrm{NADH}$, the CMP-NPs catalyze the oxidation of $\mathrm{NADH}$ to $\mathrm{NAD}^{+}$with superoxide as a byproduct under light irradiation. By switching the light on or off, a controlled interconversion of $\mathrm{NAD}^{+}$and $\mathrm{NADH}$ can be achieved, relying on the artificial antioxidant defense system based on SOD and CAT that protects the enzymatic NADHproduction mediated by GDH.

$$
\begin{aligned}
& \text { SOD: } \mathbf{2} \mathrm{O}_{2}{ }^{--}+2 \mathrm{H}^{+} \rightarrow \mathrm{O}_{2}+\mathrm{H}_{2} \mathrm{O}_{2} \\
& \text { CAT: } \mathbf{2} \mathbf{H}_{2} \mathbf{O}_{2} \rightarrow \mathrm{O}_{2}+2 \mathrm{H}_{2} \mathrm{O} \\
& \text { GDH: } \beta \text {-D-glucose }+\mathbf{N A D}^{+} \\
& \rightarrow \text { D-glucono- } \delta \text {-lactone }+\mathbf{N A D H}+\mathrm{H}^{+}
\end{aligned}
$$

Moreover, as the silica matrix is an additional barrier for diffusion, we believe any singlet oxygen that could be produced during the light-irradiation would not reach the enzymes inside the nanoreactors because of its extremely short lifetime in water $(\tau=3.1-4.5 \mu \mathrm{s}){ }^{31}$ As a result, the interior of the nanoreactors could be regarded as a "ROS safe zone" by the reactions of nanoconfined SOD/CAT and the silica barrier, enabling effective protection of GDH from ROS-mediated damages and $\mathrm{NADH}$ production.

\section{RESULTS AND DISCUSSION}

Preparation and Characterization of Enzyme-Loaded Nanoreactors. The nanoreactors containing GDH, SOD, and 


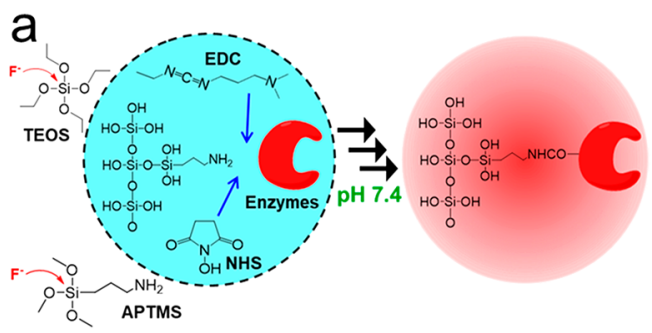

b
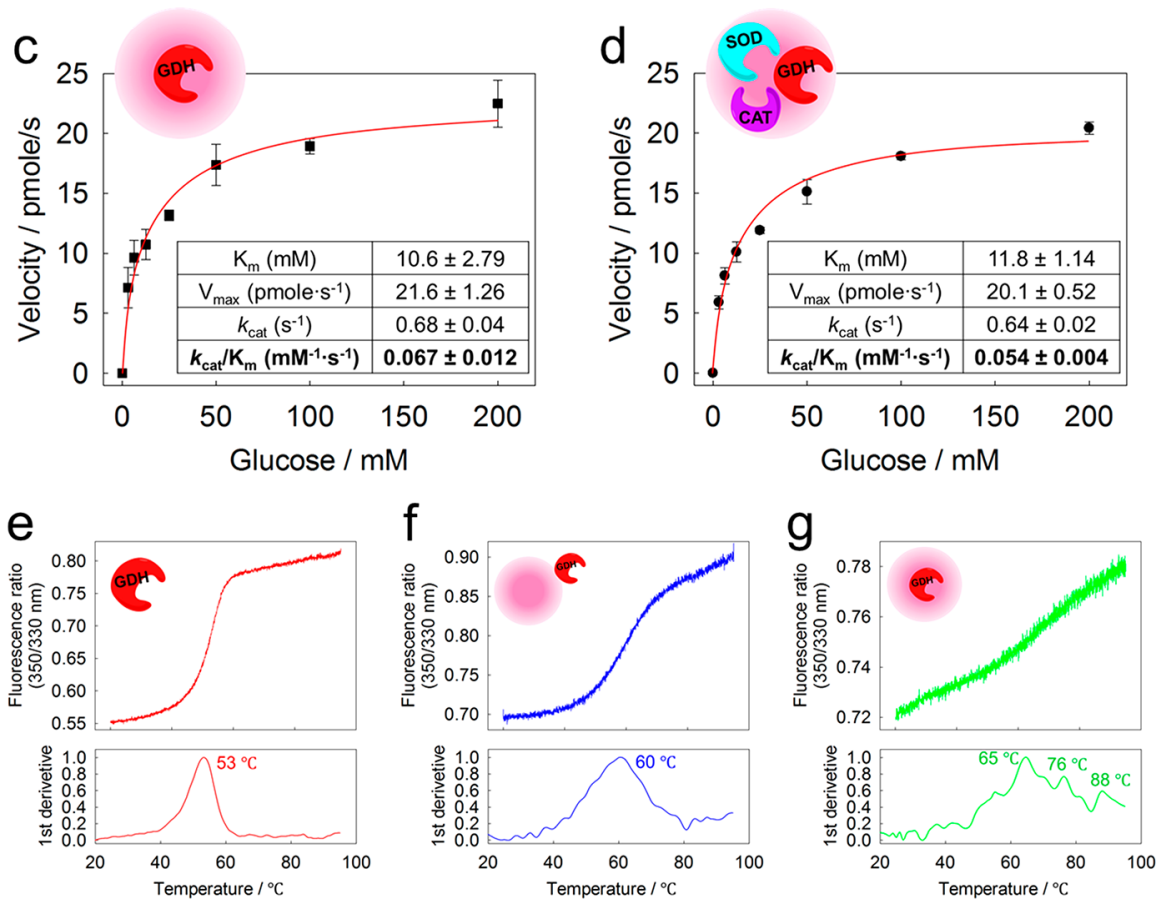

Figure 1. Preparation and characterization of nanoreactors and enzymes. (a) Fluoride-catalyzed sol-gel chemistry and one-pot loading strategy for the synthesis of the enzyme-loaded nanoreactors. (b) TEM images of GDH@nanoreactors (left) and GSC@nanoreactors (right). (c, d) Activity of GDH by Michaelis-Menten kinetics of GDH@nanoreactors and GSC@nanoreactors. (e-g) Thermal denaturation profiles of native GDH, surface-immobilized GDH, and GDH@nanoreactors determined by NanoDSF.

CAT (i.e., GSC@nanoreactors) were prepared by fluoridecatalyzed sol-gel chemistry in microemulsion (Figure 1a). Water-in-oil microemulsions were prepared with cyclohexane as a continuous phase, Triton $\mathrm{X}-100$ as an emulsifier, a buffered solution ( $\mathrm{pH} 7.4)$ as a dispersed phase, and $n$-hexanol as a second emulsifier. To the continuous phase was added tetraethyl orthosilicate (TEOS) as a silicate precursor, whereas the amphiphilic aminopropyl trimethoxysilane (APTMS) as another silicate precursor, together with $N$-hydroxysuccinimide (NHS), 1-ethyl-3-(3-(dimethylamino)propyl)carbodiimide (EDC), and the enzymes (GDH, SOD, CAT), were added to the dispersed phase. Inside the aqueous droplets, the coupling reaction between carboxyl groups of the enzymes and amino groups of the APTMS led to stable amide linkages within the silica matrix. Silica nanoreactors were readily constructed by a fluoride-catalyzed sol-gel process. This protocol enabled the preparation of enzyme-loaded nanoreactors at neutral $\mathrm{pH}$ value to keep the enzyme activity after loading to the nanoreactors. Moreover, the enzymes exhibited higher thermal and proteolytic resistance than the native enzymes. ${ }^{32,33}$ GDH-loaded nanoreactors(GDH@nanoreactors) were prepared as a control (nonantioxidant defense nanoreactor). Both nanoreactors proved colloidal stability in water after the preparation, and transmission electron microscopy (TEM) proved the spherical morphology of the nanoreactors with diameters in the range of 50-90 nm (Figure 1b). Dynamic light scattering (Figure S1) indicated diameters of ca. $205 \mathrm{~nm}$ (polydispersity index, PDI = 0.182, GDH@ nanoreactors) and $170 \mathrm{~nm}$ (PDI = 0.134, GSC@nanoreactors), respectively. Permeability of the nanoreactors that were prepared by the fluoride-catalyzed sol-gel reaction was mentioned in our previous report. ${ }^{34}$ The enzyme content of the silica nanoreactors was estimated by thermogravimetric analysis (TGA) (Figure S2). Empty nanoreactors showed ca. $20 \%$ weight loss during TGA. An additional weight loss of $11 \%$ (GDH@nanoreactors) or 21\% (GSC@nanoreactors) indicates the covalent attachment of the enzymes into the silica nanoreactors. Only 3\% additional weight loss was observed when the enzymes were loaded without covalent attachment to the silica matrix.

We determined the enzymatic reaction of the nanoreactors. Michaelis-Menten kinetics underlined the remaining activity of $\mathrm{GDH}$ in both nanoreactors (Figures 1c, d), with no significant differences in $K_{\mathrm{m}}, V_{\max }, k_{\text {cat }}$ and $k_{\text {cat }} / K_{\mathrm{m}}$ of $\mathrm{GDH}$ between GDH@nanoreactors or GSC@nanoreactors, implying the similar loaded amount of GDH in both nanoreactors. Compared to native GDH (Figure S3), there are no changes in $K_{\mathrm{m}}$ value, whereas the catalytic turnover number $\left(k_{\mathrm{cat}}\right)$ of the $\mathrm{GDH}$ in the nanoreactor is decreasing, which is possibly due to encapsulation effects. Enzymatic activity of SOD and CAT in 

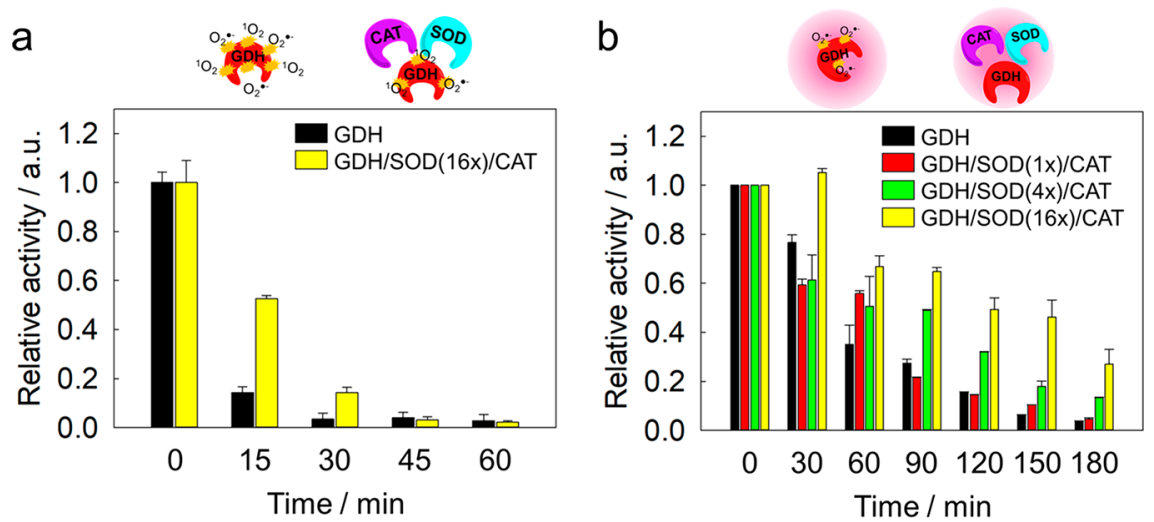

Figure 2. Oxidative stress resistance of native GDH, loaded GDH in nanoreactor without antioxidant defense systems, and loaded GDH in nanoreactor with an antioxidant defense system. Monitoring of GDH activity in the presence of ROS (generated by CMP-NPs under blue light irradiation) of (a) free GDH with (yellow) and without (black) SOD/CAT and (b) loaded GDH in a different amounts of coloaded SOD and CAT. Blue LED light $(0.84 \mathrm{~W}, 1 \mathrm{~cm}$ distance) was used as a light source.

GSC@nanoreactors was also determined by competitive reaction assays (Figures S4 and S5). Compared to the native enzymes in solution, 46 and $38 \%$ residual activity for SOD and CAT, respectively, in the nanoreactors was determined. Delayed diffusion of substrates and coenzymes by the silica barrier, conformational changes in structure, and accumulation of products in the nanoreactors might explain the decreased activity. The leakage of the enzymes from the GSC@ nanoreactors was also investigated. Negligible leakage was observed in all enzymes, which also supports covalent immobilization of enzymes to the nanoreactors (Figure S6).

Thermal stability of loaded GDH (GDH@nanoreactors) was evaluated by NanoDSF (nano differential scanning fluorimetry), which measures the thermal denaturation profile by monitoring changes in the intrinsic fluorescence of different folding states of proteins. ${ }^{35,36}$ The loaded GDH exhibited higher thermal stability than the native GDH, as indicated by the increased melting temperatures (Figure $1 \mathrm{e}-\mathrm{g}$ ). In addition, surface-immobilized GDH onto plain nanoreactors also exhibited higher stability than native GDH, but only to a lower extent compared to the encapsulated GDH, indicating an effective loading of the enzyme inside of the nanoreactors. Even though the loaded-GDH exhibited decreased activity, the increased thermal stability implies that multiple attachments to the silica matrix stabilize the tertiary structure of the enzyme. Previous reports demonstrated that immobilized proteins with multipoint attachment had increased stability against physical and chemical deactivation. ${ }^{37,38}$ This result suggested that the loaded enzyme in the nanoreactors is more durable (e.g., against higher temperature). Regarding other enzymes, native SOD already did not reveal a sharp melting by this method and was not further investigated in this paper. CAT exhibited a melting event at $55{ }^{\circ} \mathrm{C}$ in its native state; no denaturation was observed after the loading, which together with the remaining activity suggests increased thermal stability.

Proteolytic resistance of the loaded GDH in GSC@ nanoreactors was also investigated (Figure S7). Native GDH and surface-attached GDH on empty silica nanoreactors quickly lose their enzymatic activity in the presence of proteinase $\mathrm{K}$ (a proteolytic enzyme, $28.9 \mathrm{kDa}$; EC 3.4.21.64). In contrast, the encapsulated GDH inside of the nanoreactors still kept a significant degree of activity after incubation with proteinase $\mathrm{K}$. This stability against proteolysis suggests that the majority of GDH is immobilized inside the nanoreactors and is an efficient shielding by the silica shell against proteolysis.

Antioxidant Defense Activity of the Nanoreactors. We evaluated the degradation kinetics of $\mathrm{GDH}$ inside of GSC@nanoreactors in the presence of the CMP-NPs, which produced ROS under irradiation of visible light. Morphology and size distribution of CMP-NPs are described in Figure S8. ${ }^{29}$ Under the irradiation of light in solution, native GDH was rapidly lost its activity within $30 \mathrm{~min}$ in the presence of CMPNPs (Figure 2a) because of the generated superoxide and singlet oxygen. GDH, SOD, and CAT in solution were not able to effectively protect the $\mathrm{NADH}$ production. In contrast, GDH@nanoreactors exhibited significantly increased stability, which was further increased in the GSC@nanoreactors. The lifetime of GDH could be further controlled by the amount of coloaded SOD inside of the nanoreactors underlining the effect of the artificial defense system (Figure $2 b$ ). The higher stability of GDH@nanoreactor compared to GDH in the solution can be rationalized by the silica barrier of the nanoreactors, which acts as a protective layer against singlet oxygen by a delayed diffusion. Our previous report proved that a silica matrix led to a delayed diffusion of small molecules. ${ }^{33}$ As the lifetime of singlet oxygen is known to be ca. $3.1-4.5 \mu \mathrm{s}$ in water, ${ }^{31}$ it is probably deactivated before penetrating the nanoreactor. The resulting ratio of activity in each enzyme for GSC@ nanoreactors (16x the amount of SOD; Figure $2 b)$ is GDH:SOD:CAT $=1: 140: 14$ (units).

Furthermore, we found that CAT was essential for the protection of the enzyme cascade. Only using SOD or CAT did not effectively protect the GDH activity (Figure S9). Only a set of SOD/CAT showed the protection of GDH. The CAT removed the hydrogen peroxide, which is a byproduct of the SOD reaction. We believe that CAT reduced the feedback inhibition on SOD by eliminating the hydrogen peroxide.

Combination of Nanoreactors and Photocatalysts for $\mathrm{NAD}^{+} / \mathrm{NADH}$ Regeneration. To set up a light-controlled interconversion of $\mathrm{NAD}^{+}$and $\mathrm{NADH}$, we combined the GSC@nanoreactors as an $\mathrm{NADH}$ regeneration module with the CMP-NPs as an $\mathrm{NAD}^{+}$regeneration module that is only activated by visible-light irradiation (Scheme 1c). The GDH in the GSC@nanoreactors produced NADH by consuming glucose and $\mathrm{NAD}^{+}$. The CMP-NPs oxidized $\mathrm{NADH}$ to $\mathrm{NAD}^{+}$under irradiation of visible light $(460 \mathrm{~nm})$ through the production of superoxide anion. ${ }^{29}$ Thus, the interconver- 

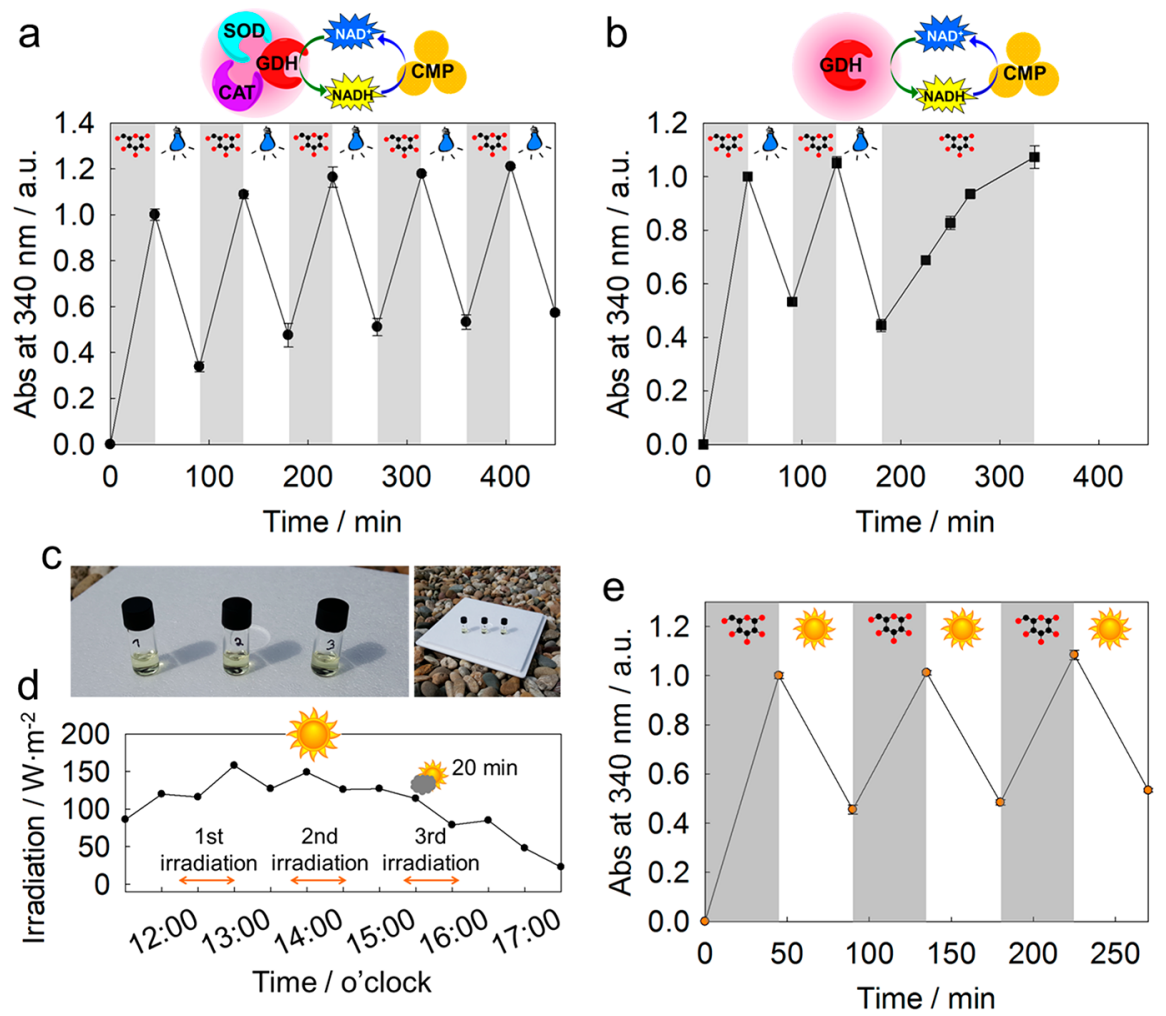

Figure 3. Recycling of $\mathrm{NAD}^{+}$and $\mathrm{NADH}$ by alternating addition of glucose in the dark and irradiation with light. Dispersions of photocatalytic CMP-NPs were combined with (a) GSC@nanoreactors or (b)GDH@nanoreactors. Each step for adding glucose at the dark condition or irradiating blue LED light $(0.84 \mathrm{~W}, 1 \mathrm{~cm}$ distance) is kept for $45 \mathrm{~min}$. (c) Experimental setting for sunlight irradiation. (d) Intensity of sunlight during the regeneration. (e) Regeneration of $\mathrm{NAD}^{+}$and $\mathrm{NADH}$ by using sunlight. For $\mathrm{a}, \mathrm{b}$, and e, increases in the absorbance mean production $\mathrm{NADH}$. Decrease in the absorbance means the production of $\mathrm{NAD}^{+}$. Initial conditions: an aqueous glucose $(2.5 \mathrm{mM})$ and $\mathrm{NAD}^{+}(5.0 \mathrm{mM})$ solutions, the same amount of glucose was added in each cycle.

sion of $\mathrm{NADH}$ and $\mathrm{NAD}^{+}$can be controlled by two different signals: glucose or light (Scheme 1c). When the antioxidant defense system is absent, overproduced superoxide would deactivate GDH. By coloading the antioxidant enzymes (SOD and $\mathrm{CAT}$ ), GDH was protected against oxidation. Morever, as SOD is located only inside the nanoreactors, $\mathrm{NAD}^{+}$production outside of the nanoreactors by the CMP-NPs was still feasible.

Controlled Recycling for NADH/NAD ${ }^{+}$Interconversion by LED Light. The controlled recycling of either NADH or $\mathrm{NAD}^{+}$regeneration was carried out by a combination of the two modules, the GSC@nanoreactors and the photoactive CMP-NPs. Glucose and $\mathrm{NAD}^{+}$are converted to gluconic acid and NADH by GDH in the GSC@nanoreactors in the dark. When irradiated, the photo-oxidation regenerated $\mathrm{NAD}^{+}$ mediated by the CMP-NPs (blue LED lamp, $0.84 \mathrm{~W}$ ). The interconversion of $\mathrm{NADH} / \mathrm{NAD}^{+}$was repeated for five cycles (Figure 3a) and did not show significant changes in the reaction velocity of each cycle (about $45 \mathrm{~min}$ ), which means the protection of GDH was successful by coloading both SOD and CAT. In contrast, the recycling with single-loaded GDH@ nanoreactors could only be repeated 3 times with decreased activity because of the oxidation of GDH by reaction with ROS (Figure 3b).

To further explore the availability of sunlight for photocatalytic reactions, we performed the interconversion of $\mathrm{NAD}^{+}$ and $\mathrm{NADH}$ in sun light as green energy source. For the recycling study, GSC@nanoreactors and CMP-NPs were mixed and irradiated on a sunny day in late summer in Mainz (Germany). The sunlight intensity during the day was monitored (Figure 3c, d) and three repeating cycles for the regeneration were conducted during daylight. Figure $3 \mathrm{e}$ shows the cycles after the addition of glucose in the dark and placing the reaction again in the sunlight. It has to be mentioned that the sunlight increased the temperature of the dispersion (depending on the weather conditions); however, the temperature variation did not interfere with the recycling reaction, as the encapsulated enzymes proved high-temperature resistance (see above). When the vials were placed in the shade or only indirect sunlight, no regeneration of $\mathrm{NAD}^{+}$was observed (Figure S10).

Antioxidation Activity of the Nanoreactors. Above, we demonstrated the antioxidant defense strategy to preserve the enzyme activity of GDH inside of the nanoreactors. When $\mathrm{NADH}$ is produced inside of the nanoreactors, it might diffuse to the continuous phase (outside of the nanoreactors). In the solution, $\mathrm{NADH}$ can act as an additional ROS scavenger (Figure 1d), i.e., we assumed that our system could also exhibit an antioxidant activity outside of the nanoreactors. To demonstrate this, we added Amplex red as an oxidation probe to the dispersion of GDH/SOD/CAT@nanoreactors and CMP-NPs. ROS can oxidize both NADH and Amplex red (Figure 4a). In the absence of $\mathrm{NADH}$, Amplex red was quickly oxidized to resorufin by superoxide when irradiated with visible light, which was detected by a change in fluorescence (Figure $4 \mathrm{~b}$ ). In contrast, when $\mathrm{NADH}$ was produced by GDH, i.e., by simultaneous addition of glucose and irradiation, the oxidation of Amplex red was inhibited as it competed with $\mathrm{NADH}$ (Figure $4 \mathrm{~b}$ ). As the resulting $\mathrm{NAD}^{+}$can be reduced by $\mathrm{GDH}$ 

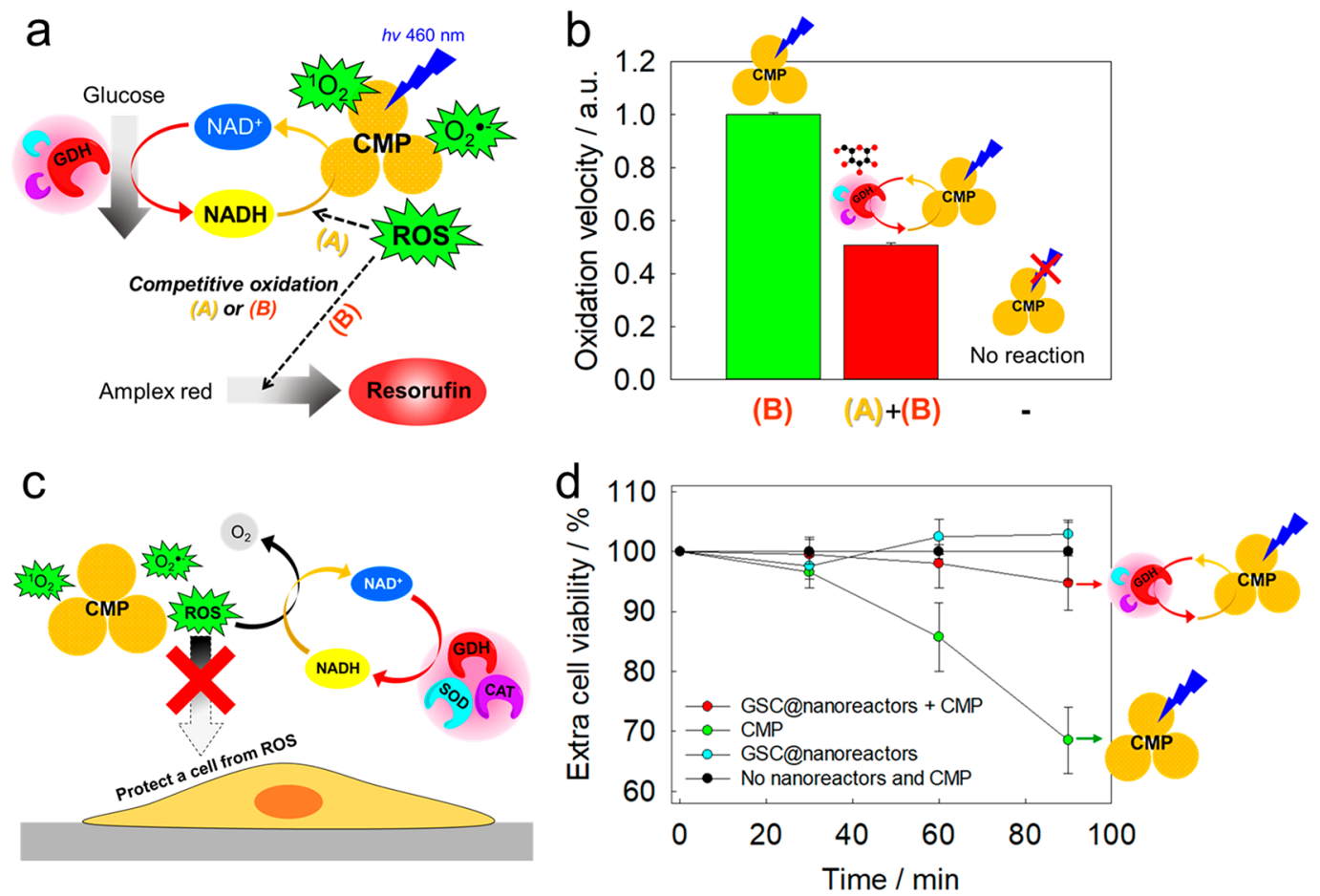

Figure 4. Antioxidant defense strategy for the exterior area. (a) Regenerated and secreted NADH from the nanoreactors works as a scavenger against superoxide for the antioxidative defense at the exterior of the nanoreactors. Under $\mathrm{NAD}^{+} / \mathrm{NADH}$ regeneration by adding glucose or photoactivation of CMPs, NADH (pathway A: producing $\mathrm{NAD}^{+}$) and Amplex red (pathway B: producing resorufin) are competitively oxidized by superoxide. (b) Antioxidative activity of GSC@nanoreactors and CMP-NPs toward the exterior environment by consuming glucose and irradiation. The assay condition was constituted by mixing Amplex red $(30.0 \mu \mathrm{M})$ and NADH $(0.15 \mu \mathrm{M})$. Without irradiation, it could not lead the production of resorufin. Blue LED light $(0.84 \mathrm{~W}, 1 \mathrm{~cm}$ distance) was used as a light source. (c) Scheme of cycto-protective function of GSC@nanoreactors vs CMP-mediated oxidative stress. (d) Relative cell-viability measured by CCK-8 assay of MDA-MB-231 cells in the presence of CMP-NPs and GSC@nanoreactors under light irradiation compared to dark conditions. Effective cell protection of the GSC@nanoreactors was achieved by enzymatic $\mathrm{NADH}$ production.

reaction, continuous recycling of $\mathrm{NAD}^{+} / \mathrm{NADH}$ was achieved (note: without irradiation of light, no oxidation was detected).

Finally, the antioxidative cell protection of GSC@nanoreactors was studied (Figure 4c). When MDA-MB-231 as a model cell line was treated with GSC@nanoreactors and CMP-NPs and irradiated with visible light $(460 \mathrm{~nm})$, the cells were successfully protected from the CMP-mediated oxidation because of the scavenging effect of NADH (Figure $4 \mathrm{~d}$ ). The GSC@nanoreactors and the CMP-NPs can continuously regenerate the $\mathrm{NAD}^{+} / \mathrm{NADH}$, and thus this antioxidation behavior would be sustainable by only supplying glucose and oxygen. In contrast, when cells were treated with photocatalytic CMP-NPs and irradiated with light, significant cell death was observed (note: relatively low cytotoxicity was observed for CMP-NPs in the dark). The combination of the GSC@nanoreactors together with photocatalytic systems was able to protect living cells and might be expanded to other biological systems, e.g., artificial cellular organelles.

\section{CONCLUSIONS}

An artificial, bioinspired antioxidant defense system based on the combination of three enzymes (GDH, SOD, CAT) which were covalently loaded into silica nanoreactors, was developed. When SOD and CAT were confined inside of the nanoreactors, they proved effective protection against oxidation of $\mathrm{GDH}$. When the substrate glucose was added, NADH was produced by GDH. A further combination with photocatalytic nanoparticles (CMP-NPs) reproduced the $\mathrm{NAD}^{+}$when irradiated with light but also produced superoxide. By protecting GDH against superoxide, a recyclable and controllable $\mathrm{NAD}^{+} / \mathrm{NADH}$ regeneration was achieved. The $\mathrm{NAD}^{+} /$ $\mathrm{NADH}$ redox pair is a key coenzyme for energy storage and redox reactions in living organisms and artificial biosystems. The oxidoreductase family is one of the largest classes of all enzymes $(\sim 25 \%)$, and it relies on the $\mathrm{NAD}^{+} / \mathrm{NADH}$ redox pair. Thus, living cells use their regenerative pathways to manage efficient metabolism. Moreover, the regenerative uses of $\mathrm{NAD}^{+}$orNADH for in vitro bioreactions is economically feasible. A major advantage of the antioxidant-loaded nanoreactors could increase the sustained availability of enzymes. Our strategy might be a versatile tool for enzyme applications for in vitro biological reactors, enzyme-controlled organic reactions, or prototypes of artificial organelles or building blocks. We additionally demonstrated that sunlight could be utilized for $\mathrm{NAD}^{+}$regeneration. This concept might open up new possibilities of bioenergy regeneration and photosynthesis by green energy sources. Our antioxidant nanoreactors also allow safe availability of photocatalysis into biological systems.

\section{ASSOCIATED CONTENT}

\section{Supporting Information}

The Supporting Information is available free of charge at https://pubs.acs.org/doi/10.1021/acsami.0c05588.

Materials and methods; size measurement of nanoreactors (Figure S1); TGA of nanoreactors (Figure S2); Michaelis-Menten kinetics of native GDH (Figure S3); 
principle of enzymatic assay and standard curve of SOD reaction (Figure S4); principle of enzymatic assay and standard curve of CAT reaction (Figure S5); enzyme leakage of GSC@nanoreactors (Figure S6); proteinase K resistance of $\mathrm{GDH}$ and nanoreactors (Figure S7); size and TEM image of CMP-NPs (Figure S8); oxidative stress resistance of native GDH/SOD and native GDH/ CAT (Figure S9); regeneration of $\mathrm{NAD}^{+}$and $\mathrm{NADH}$ in the shade (Figure S10) (PDF)

\section{AUTHOR INFORMATION}

\section{Corresponding Authors}

Frederik R. Wurm - Max Planck Institute for Polymer Research, Mainz D-55128, Germany; 이잉.org/00000002-6955-8489; Email: wurm@mpip-mainz.mpg.de

Katharina Landfester - Max Planck Institute for Polymer Research, Mainz D-55128, Germany; 이이.org/00000001-9591-4638; Email: landfester@mpip-mainz.mpg.de

\section{Authors}

Seong-Min Jo - Max Planck Institute for Polymer Research, Mainz D-55128, Germany

Kai A. I. Zhang - Max Planck Institute for Polymer Research, Mainz D-55128, Germany; Department of Materials Science, Fudan University, Shanghai 200433, China; (o orcid.org/ 0000-0003-0816-5718

Complete contact information is available at: https://pubs.acs.org/10.1021/acsami.0c05588

\section{Author Contributions}

The manuscript was written through the contributions of all authors. All authors have approved the final version of the manuscript.

Notes

The authors declare no competing financial interest.

\section{ACKNOWLEDGMENTS}

This work is part of the MaxSynBio consortium, which was jointly funded by the Federal Ministry of Education and Research of Germany and the Max Planck Society. We appreciate Dr. Beatriz C. Ma (MPIP) for preparing photocatalytic nanoparticles.

\section{ABBREVIATIONS}

APTMS, aminopropyl trimethoxysilane; CAT, catalase; CMPs, conjugated microporous polymers; EDC, 1-ethyl-3-(3(dimethylamino)propyl)carbodiimide; GDH, glucose dehydrogenase; $\mathrm{NAD}$, nicotinamide adenine dinucleotide; $\mathrm{NAD}^{+}$, oxidized form of nicotinamide adenine dinucleotide; $\mathrm{NADH}$, reduced form of nicotinamide adenine dinucleotide; NHS, $\mathrm{N}$ hydroxysuccinimide; NPs, nanoparticles; PDI, polydispersity index; ROS, reactive oxygen species; TEOS, tetraethyl orthosilicate

\section{REFERENCES}

(1) Birben, E.; Sahiner, U. M.; Sackesen, C.; Erzurum, S.; Kalayci, O. Oxidative Stress and Antioxidant Defense. World Allergy Organ. J. 2012, 5 (1), 9-19.

(2) Marengo, B.; Nitti, M.; Furfaro, A. L.; Colla, R.; Ciucis, C. D.; Marinari, U. M.; Pronzato, M. A.; Traverso, N.; Domenicotti, C. Redox Homeostasis and Cellular Antioxidant Systems: Crucial Players in Cancer Growth and Therapy \%J Oxidative Medicine and Cellular Longevity. Oxid. Med. Cell. Longevity 2016, 2016, 16.
(3) Ighodaro, O. M.; Akinloye, O. A. First line defence antioxidantssuperoxide dismutase (SOD), catalase (CAT) and glutathione peroxidase (GPX): Their fundamental role in the entire antioxidant defence grid. Alexandria J. Med. 2018, 54 (4), 287-293.

(4) Vernekar, A. A.; Sinha, D.; Srivastava, S.; Paramasivam, P. U.; D'Silva, P.; Mugesh, G. An antioxidant nanozyme that uncovers the cytoprotective potential of vanadia nanowires. Nat. Commun. 2014, 5, 5301.

(5) Li, W.; Liu, Z.; Liu, C.; Guan, Y.; Ren, J.; Qu, X. Manganese Dioxide Nanozymes as Responsive Cytoprotective Shells for Individual Living Cell Encapsulation. Angew. Chem., Int. Ed. 2017, 56 (44), 13661-13665.

(6) Huang, Y.; Liu, Z.; Liu, C.; Ju, E.; Zhang, Y.; Ren, J.; Qu, X. SelfAssembly of Multi-nanozymes to Mimic an Intracellular Antioxidant Defense System. Angew. Chem., Int. Ed. 2016, 55 (23), 6646-6650.

(7) Wang, H.; Xu, X.; Chen, R.; Zhao, J.; Cui, L.; Sheng, G.; Shi, Q.; Wong, S.-C.; Yin, J. Bioinspired Antioxidant Defense System Constructed by Antioxidants-Eluting Electrospun F127-Based Fibers. ACS Appl. Mater. Interfaces 2017, 9 (44), 38313-38322.

(8) Singh, N.; Savanur, M. A.; Srivastava, S.; D'Silva, P.; Mugesh, G. A Redox Modulatory Mn3O4 Nanozyme with Multi-Enzyme Activity Provides Efficient Cytoprotection to Human Cells in a Parkinson's Disease Model. Angew. Chem., Int. Ed. 2017, 56 (45), 14267-14271.

(9) Singh, N.; Savanur, M. A.; Srivastava, S.; D'Silva, P.; Mugesh, G. A manganese oxide nanozyme prevents the oxidative damage of biomolecules without affecting the endogenous antioxidant system. Nanoscale 2019, 11 (9), 3855-3863.

(10) Chen, Y.-P.; Chen, C.-T.; Hung, Y.; Chou, C.-M.; Liu, T.-P.; Liang, M.-R.; Chen, C.-T.; Mou, C.-Y. A New Strategy for Intracellular Delivery of Enzyme Using Mesoporous Silica Nanoparticles: Superoxide Dismutase. J. Am. Chem. Soc. 2013, 135 (4), $1516-1523$.

(11) Einfalt, T.; Witzigmann, D.; Edlinger, C.; Sieber, S.; Goers, R.; Najer, A.; Spulber, M.; Onaca-Fischer, O.; Huwyler, J.; Palivan, C. G. Biomimetic artificial organelles with in vitro and in vivo activity triggered by reduction in microenvironment. Nat. Commun. 2018, 9 (1), 1127.

(12) Axthelm, F.; Casse, O.; Koppenol, W. H.; Nauser, T.; Meier, W.; Palivan, C. G. Antioxidant Nanoreactor Based on Superoxide Dismutase Encapsulated in Superoxide-Permeable Vesicles. J. Phys. Chem. B 2008, 112 (28), 8211-8217.

(13) Onaca, O.; Hughes, D. W.; Balasubramanian, V.; Grzelakowski, M.; Meier, W.; Palivan, C. G. SOD Antioxidant Nanoreactors: Influence of Block Copolymer Composition on the Nanoreactor Efficiency. Macromol. Biosci. 2010, 10 (5), 531-538.

(14) Chang, F.-P.; Chen, Y.-P.; Mou, C.-Y. Intracellular Implantation of Enzymes in Hollow Silica Nanospheres for Protein Therapy: Cascade System of Superoxide Dismutase and Catalase. Small 2014, 10 (22), 4785-4795.

(15) Larrañaga, A.; Isa, I. L. M.; Patil, V.; Thamboo, S.; Lomora, M.; Fernández-Yague, M. A.; Sarasua, J.-R.; Palivan, C. G.; Pandit, A. Antioxidant functionalized polymer capsules to prevent oxidative stress. Acta Biomater. 2018, 67, 21-31.

(16) Nam, D. H.; Kuk, S. K.; Choe, H.; Lee, S.; Ko, J. W.; Son, E. J.; Choi, E.-G.; Kim, Y. H.; Park, C. B. Enzymatic photosynthesis of formate from carbon dioxide coupled with highly efficient photoelectrochemical regeneration of nicotinamide cofactors. Green Chem. 2016, 18 (22), 5989-5993.

(17) Liu, J.; Antonietti, M. Bio-inspired NADH regeneration by carbon nitride photocatalysis using diatom templates. Energy Environ. Sci. 2013, 6 (5), 1486-1493.

(18) Wu, H.; Tian, C.; Song, X.; Liu, C.; Yang, D.; Jiang, Z. Methods for the regeneration of nicotinamide coenzymes. Green Chem. 2013, 15 (7), 1773-1789.

(19) Wang, X.; Saba, T.; Yiu, H. H. P.; Howe, R. F.; Anderson, J. A.; Shi, J. Cofactor $\mathrm{NAD}(\mathrm{P}) \mathrm{H}$ Regeneration Inspired by Heterogeneous Pathways. Chem. 2017, 2 (5), 621-654.

(20) Kim, J. H.; Nam, D. H.; Park, C. B. Nanobiocatalytic assemblies for artificial photosynthesis. Curr. Opin. Biotechnol. 2014, 28, 1-9. 
(21) Lin, S.-J.; Guarente, L. Nicotinamide adenine dinucleotide, a metabolic regulator of transcription, longevity and disease. Curr. Opin. Cell Biol. 2003, 15 (2), 241-246.

(22) Shaik, F.; Peer, I.; Jain, P. K.; Amirav, L. Plasmon-Enhanced Multicarrier Photocatalysis. Nano Lett. 2018, 18 (7), 4370-4376.

(23) Mifsud, M.; Gargiulo, S.; Iborra, S.; Arends, I. W. C. E.; Hollmann, F.; Corma, A. Photobiocatalytic chemistry of oxidoreductases using water as the electron donor. Nat. Commun. 2014, 5, 3145.

(24) Lv, M.; Sun, X.; Wei, S.; Shen, C.; Mi, Y.; Xu, X. Ultrathin Lanthanum Tantalate Perovskite Nanosheets Modified by Nitrogen Doping for Efficient Photocatalytic Water Splitting. ACS Nano 2017, 11 (11), 11441-11448.

(25) Brown, K. A.; Wilker, M. B.; Boehm, M.; Hamby, H.; Dukovic, G.; King, P. W. Photocatalytic Regeneration of Nicotinamide Cofactors by Quantum Dot-Enzyme Biohybrid Complexes. ACS Catal. 2016, 6 (4), 2201-2204.

(26) Marzo, L.; Pagire, S. K.; Reiser, O.; König, B. Visible-Light Photocatalysis: Does It Make a Difference in Organic Synthesis? Angew. Chem., Int. Ed. 2018, 57 (32), 10034-10072.

(27) Lee, S. H.; Choi, D. S.; Kuk, S. K.; Park, C. B. Photobiocatalysis: Activating Redox Enzymes by Direct or Indirect Transfer of Photoinduced Electrons. Angew. Chem., Int. Ed. 2018, 57 (27), 7958-7985.

(28) Wondraczek, L.; Tyystjärvi, E.; Méndez-Ramos, J.; Müller, F. A.; Zhang, Q. Shifting the Sun: Solar Spectral Conversion and Extrinsic Sensitization in Natural and Artificial Photosynthesis. Adv. Sci. 2015, 2 (12), 1500218.

(29) Ma, B. C.; Caire da Silva, L.; Jo, S.-M.; Wurm, F. R.; Bannwarth, M.; Zhang, K. A. I.; Sundmacher, K.; Landfester, K. Polymer-based module for $\mathrm{NAD}^{+}$regeneration with visible light. ChemBioChem 2019, 20 (20), 2593-2596.

(30) Lin, S.; Sun, S.; Wang, K.; Shen, K.; Ma, B.; Ren, Y.; Fan, X. Bioinspired Design of Alcohol Dehydrogenase@nano TiO2Microreactors for Sustainable Cycling of $\mathrm{NAD}^{+} / \mathrm{NADH}$ Coenzyme. Nanomaterials 2018, 8 (2), 127.

(31) Wilkinson, F.; Helman, W. P.; Ross, A. B. Rate Constants for the Decay and Reactions of the Lowest Electronically Excited Singlet State of Molecular Oxygen in Solution. An Expanded and Revised Compilation. J. Phys. Chem. Ref. Data 1995, 24 (2), 663-677.

(32) Ellerby, L. M.; Nishida, C. R.; Nishida, F.; Yamanaka, S. A.; Dunn, B.; Valentine, J. S.; Zink, J. I. Encapsulation of proteins in transparent porous silicate glasses prepared by the sol-gel method. Science 1992, 255 (5048), 1113-1115.

(33) Jo, S.-M.; Wurm, F. R.; Landfester, K. Biomimetic Cascade Network between Interactive Multicompartments Organized by Enzyme-Loaded Silica Nanoreactors. ACS Appl. Mater. Interfaces 2018, 10 (40), 34230-34237.

(34) Jo, S.-M.; Wurm, F. R.; Landfester, K. Oncolytic Nanoreactors Producing Hydrogen Peroxide for Oxidative Cancer Therapy. Nano Lett. 2020, 20 (1), 526-533.

(35) Strutz, W. Exploring Protein Stability by NanoDSF. Biophys. J. 2016, 110 (3, Suppl. 1), 393a.

(36) Magnusson, A. O.; Szekrenyi, A.; Joosten, H.-J.; Finnigan, J.; Charnock, S.; Fessner, W.-D. nanoDSF as screening tool for enzyme libraries and biotechnology development. FEBS J. 2019, 286 (1), 184-204.

(37) Matthijs, G.; Schacht, E. Comparative study of methodologies for obtaining $\beta$-glucosidase immobilized on dextran-modified silica. Enzyme Microb. Technol. 1996, 19 (8), 601-605.

(38) Klibanov, A. M. Enzyme stabilization by immobilization. Anal. Biochem. 1979, 93, 1-25. 